\title{
Cyclodextrin-ionic liquid polyurethanes for application in drinking water treatment
}

\author{
TJ Malefetse, BB Mamba*, RW Krause and MM Mahlambi \\ University of Johannesburg, Department of Chemical Technology, PO Box 17011, Doornfontein 2028, South Africa
}

\begin{abstract}
The prevalence of toxic contaminants in water remains a huge challenge for water-supplying companies and municipalities. Both organic and inorganic (especially heavy metals) pollutants are often present in water distribution networks. The presence of these contaminants in drinking water poses a major risk to human health. Organic and inorganic pollutants often co-occur in drinking water networks. However, at present there is no water treatment intervention that simultaneously removes both organic and inorganic pollutants from water to desirable levels. In our laboratories, recent studies have shown that both functionalised and un-functionalised cyclodextrin (CD) polymers are capable of removing organic pollutants from water, with the functionalised CD polymers showing an enhanced absorption capability. Ionic liquids (ILs), on the other hand, have been reported to absorb heavy metals from aqueous media. In this paper, we report on the synthesis of several cyclodextrin-ionic liquid (CD-IL) polymers, a dual system capable of removing both organic and inorganic pollutants from water. This system has been tested and has proved to possess excellent capabilities for the removal of model pollutants such as $p$-nitrophenol (PNP), 2,4,6-trichlorophenol (TCP) and chromium $\left(\mathrm{Cr}^{6+}\right)$ from aqueous media.
\end{abstract}

Keywords: organic and inorganic pollutants, CD polymers, ionic liquids, CD-IL polymers

\section{Introduction}

Organic and inorganic water pollutants pose a major threat to human health even when present at low concentrations. For instance, because organic pollutants persist in the environment for long periods of time, they can be absorbed by plants thereby finding their way to poisoning the food chains of living organisms (Oleszczuk et al., 2004). They have also been linked to adverse human effects such as cancer, nervous system damage, reproductive disorders, as well as the disruption of the immune system (EPA, 2006). Also, organic contaminants released in one part of the world can be transported globally via oceans and atmospherically, and their effects can be felt in regions which are distant from where they originate.

On the other hand, inorganic pollutants, particularly heavy metals such as lead, cadmium, mercury and silver, have been reported to be toxic and even lethal to the human body, especially the central nervous system (Morales et al., 1999). These metals have a great affinity for sulphur and hence attack the sulphur bonds of enzymes causing them to malfunction (Lewis et al., 2001; Morales et al., 1999). One of the primary sources of heavy metal pollution in developing countries is industrial discharge. Taking into consideration the toxicity and the bioaccumulation of organic and inorganic pollutants, even in drinking water networks, a technique that can simultaneously remove both inorganic and organics from water needs to be developed. In this paper, we thus report on a technique that utilises cyclodextrin-ionic liquid (CD-IL) polymers for the simultaneous quenching of these pollutants from drinking water.

Cyclodextrins (CDs), first discovered by Villiers in 1891, are cyclic oligomers formed by the enzymatic hydrolysis of

\footnotetext{
* To whom all correspondence should be addressed.

용 +2711 559-6516; fax: +2711 559-6425;

e-mail: bmamba@uj.ac.za

Received 16 May 2009; accepted in revised form 10 August 2009.
}

starch by Bacillus macerans (Szeitjli, 1998). The three most commonly known CDs contain $6(\alpha), 7(\beta)$ and $8(\gamma)$ glucose units which are linked together by $\alpha-(1,4)$ linkages (Bender and Komiyana, 1978). Liu et al. (2003) reported that CDs have a non-polar cavity which provides a micro-environment for the encapsulation of non-polar, low molecular weight compounds (formation of an inclusion complex) (Scheme 1).

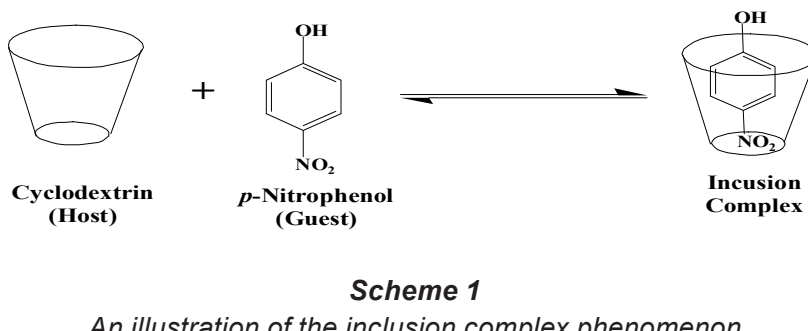

However, CDs are slightly soluble in water which limits their sole application for water treatment purposes. This necessitates the functionalisation and, in particular, polymerisation of the parental CDs with suitable bi-functional linkers such as hexamethylene diisocyanate (HDI) and toluene diisocyanate (TDI) to make them insoluble ( $\mathrm{Li}$ and Ma, 1999). These waterinsoluble cyclodextrin polymers have been successfully synthesised in our laboratories and have been found to be effective in the absorption of organic pollutants at very low $\left(\mathrm{ng} \cdot \ell^{-1}\right)$ concentrations (Mhlanga et al., 2007; Mamba et al., 2007). Also, mono-functionalised insoluble CD polymers were found to be effective in the removal of toxic phenolic compounds from the aqueous media at very low concentrations (Mamba et al., 2007). Trichloroethylene was removed to non-detectable levels whilst PNP was removed from a $10 \mathrm{mg} \cdot \ell^{-1}$ spiked water sample with a removal efficiency of $99 \%$ (Salipira et al., 2007). During functionalisation, incorporation of specific functional groups can decrease the solubility of CDs, increase their stability in 
the presence of light, heat and oxidising conditions, or decrease their volatility (Szeitjli, 1998; Harada, 1997).

Ionic liquids (ILs) have aroused increasing interest for their promising role as alternative media for volatile solvents (Wei et al., 2003). ILs are defined as salts with a low melting point composed of organic cations and mostly inorganic anions such as $\mathrm{Cl}^{-}, \mathrm{Br},\left[\mathrm{PF}_{6}\right]^{-}$and $\left[\mathrm{BF}_{4}\right]^{-}$(see Fig. 1). Besides anions such as hexafluorophosphate, tetrafluoroborate and halide anions, other common inorganic anions that are used for the preparation of ILs are $\left[\mathrm{SbF}_{6}\right]^{-}$and $\left[\left(\mathrm{CF}_{3} \mathrm{SO}_{2}\right)_{2} \mathrm{~N}\right]^{-}$. The anion need not necessarily be inorganic; ILs possessing organic anions such as alkylsulphate, tosylate and methanesulfonate are known. The most common types of organic cations are the imidazolium and pyridinium ions (Pereiro et al., 2007). Other less common cations include ammonium, phosphonium, pyrrolidinium and sulphonium cations.

Ferreira et al. (2000) reported that the forces of attraction between the cation and the anion are not sufficiently strong enough to hold them together as solids at ambient temperatures hence it is possible, by proper choice of starting material, to synthesise ionic liquids that are liquid at or below room temperature. ILs possess unique physical properties such as negligible vapour pressure, an ability to dissolve a wide range of organic and inorganic material, high thermal stability (for example, some ILs are liquid at $400^{\circ} \mathrm{C}$, while others are liquid at $-96^{\circ} \mathrm{C}$ ) as well as variable viscosity and miscibility with water and other organic solvents (Liu et al., 2003; Liu et al., 2005; Welton, 1999). However, the most important property of ionic liquids that has been exploited in this study is their ability to extract metals from aqueous media (Cruz, 2000; Liu et al., 2005). In this regard, Visser et al. (2002) have reported the successful extraction of cadmium $\left(\mathrm{Cd}^{2+}\right)$ and mercury $\left(\mathrm{Hg}^{2+}\right)$ from an aqueous medium using ionic liquids via the formation of an IL-metal complex.

Since water-insoluble CD polymers have demonstrated the ability to absorb organic pollutants from water even at ng $\ell^{-1}$ levels and on the other hand ILs are able to remove metal ions<smiles>[R]N1C=CN([R])C1</smiles>

$\mathrm{R}, \mathrm{R}$--dialkylimidazolium chloride

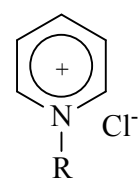

R-alkylpyridinium chloride

Figure 1

Examples of ionic liquids

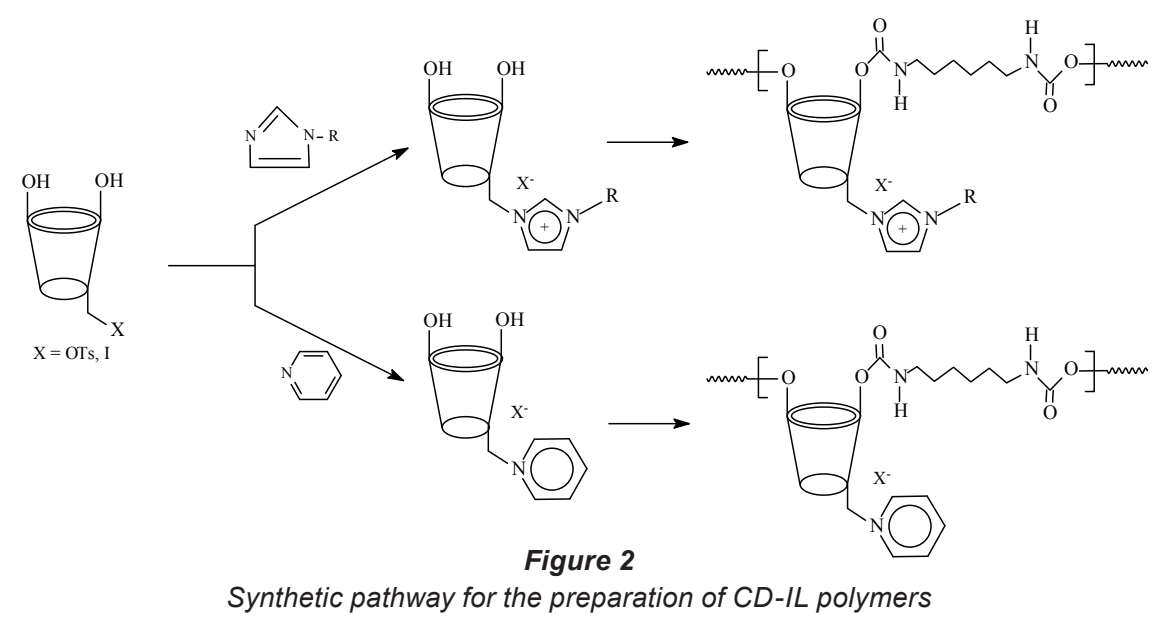


spectra were recorded at $300 \mathrm{MHz}$ using a Varian Gemini 2000 spectrometer. Proton and carbon chemical shifts are reported in $\mathrm{mg} \cdot \ell^{-1}$ using the residual signal dimethyl sulphoxide (DMSO- $\left.{ }_{\mathrm{d} 6}\right)$ $\left(\delta=2.49\right.$ for ${ }^{1} \mathrm{H}$ and 39.50 for $\left.{ }^{13} \mathrm{C}\right)$ or trimethyl silane (TMS) $(\delta$ $=0)$ as an internal reference. For ultraviolet (UV) experiments, a UV-Visible Cary 50 Spectrophotometer was used for the collection of data. A Varian CP-3800 gas chromatograph ion-trap Saturn 2000 Mass Spectrometer was used for the quantification of organic pollutants. The GC was equipped with a Chrompac $\mathrm{CP}$ Sil $8 \mathrm{CB}, 30 \mathrm{~m} \times 0.25 \mathrm{~mm}$ column with an internal diameter of $0.25 \mu \mathrm{m}$. A Varian Spectr Atomic Absorption 10 flame spectrophotometer (AAS) was used for the analysis of heavy metal ions. The AAS wavelength was set at $357.9 \mathrm{~nm}$ for the analysis of $\mathrm{Cr}^{6+}$ and a flame of air/acetylene was employed.

\section{Synthesis of CD-IL complexes}

The CD-IL complexes were synthesised from CDOTs and CDI. Due to the ease of formation of the mono-tosylate and ease with which the tosyl leaving group can be replaced, mono-tosylated cyclodextrins are generally good precursors for derivatives of CDs (Zhong et al., 1998). To prepare the CD-IL derivatives, an alkyl imidazole (10 molar equiv.) was added drop-wise to a stirred solution of either $\beta$-CDI or $\beta$-CDOTs $(0.88 \mathrm{mmol})$ dissolved in anhydrous DMF $(40 \mathrm{~m} \ell)$. Stirring was continued at elevated temperatures $\left(80^{\circ} \mathrm{C}\right.$ and $90^{\circ} \mathrm{C}$ for $\beta$-CDI an $\beta$-CDOTs, respectively) under nitrogen for a further $24 \mathrm{~h}$. After cooling to room temperature, acetone $(25 \mathrm{~m} \ell)$ was added to precipitate the product. The reaction mixture was then stirred for 30 $\mathrm{min}$. Evaporation of organic solvents using a rotary evaporator produced a white solid. This solid was finally dissolved in deionised water $(50 \mathrm{~m} \ell)$ and precipitated by the addition of acetone $(200 \mathrm{~m} \ell)$. The precipitate was filtered off and dried under vacuum to yield a white powdery solid.

\section{Synthesis of CD-IL polymers}

The CD-IL complexes in the above section (both tosylate and iodide derivatives) were reacted with bi-functional diisocyanate linkers, hexamethylene diisocyanate (HDI) and toluene-2,4diisocyanate (TDI), to produce CD-IL polymers. Typically, the $\beta$-CD-IL complex $(0.88 \mathrm{mmol})$ was dissolved in DMF $(20 \mathrm{~m} \ell)$ and the bi-functional linker was added drop-wise to the reaction mixture. The solution was allowed to react at $75^{\circ} \mathrm{C}$ for 18 to $24 \mathrm{~h}$ with constant stirring. The polymerisation reaction was monitored by IR spectroscopy. The completion of the polymerisation was confirmed by the total disappearance of the isocyanate peak at $2270 \mathrm{~cm}^{-1}$ after 18 to $24 \mathrm{~h}$ (Li and Ma, 1999). The reaction mixture was then precipitated by the addition of acetone $(100 \mathrm{~m} \ell)$. The solid formed was then left to settle in acetone for 10 min to allow for the removal of residual DMF from the polymers. To remove traces of DMF which might still be present, the polymers were filtered and washed with copious amounts of acetone $(100 \mathrm{~m} \ell)$. The polymers were then dried overnight under reduced pressure.

The polymers under investigation were derived from the tosylate and iodide derivatives of $\beta$-cyclodextrin methyl imidazolium, $\beta$-cyclodextrin butyl imidazolium and $\beta$-cyclodextrin pyridinium precursors.

Preparation of para-nitrophenol (PNP), 2,4,6-trichlorophenol (TCP) and $\mathrm{Cr}^{6+}$ standards

Potassium dichromate, $p$-nitrophenol and 2,4,6-trichlorophenol were purchased from suppliers and used without further purification. PNP standards of 2, 5, 10, 15 and $20 \mu \mathrm{g} \cdot \ell^{-1}$ were prepared and used to test the absorption efficiencies of the polymers in the absorption of organic pollutants from water. UV-visible spectroscopy was used to determine the amount of the pollutant absorbed by the polymers. A $10 \mathrm{~mm}$ cuvette cell was used as a sample holder. For the analysis of TCP, standards of $1,2,5,10,15$ and $20 \mu \mathrm{g} \cdot \ell^{-1}$ were prepared.

Gas chromatograph-mass spectrometry (GC-MS) was used for the analysis of TCP absorbed by the polymers; 2,4,6-trichlorophenol $\left(10 \mathrm{mg} \cdot \ell^{-1}\right)$ was passed through the polymers and the filtrate was analysed for the residual amount of TCP using GC-MS. Solid-phase extraction (SPE) was employed to extract TCP from the filtrate. The extractant from SPE analysis was concentrated to about $2 \mathrm{~m} \ell$ by bubbling through nitrogen gas.

To analyse for the amount of $\mathrm{Cr}^{6+}$ absorbed by the polymers, atomic absorption spectrophotometer (AAS) was employed. $\mathrm{Cr}^{6+}$ standards of 2, 4, 5, 6 and $8 \mu \mathrm{g} \cdot \ell^{-1}$ were prepared and used to determine the absorption efficiencies of the polymers. $\mathrm{A} \mathrm{Cr}^{6+}$ concentration of $5 \mathrm{mg} \cdot \ell^{-1}$ was chosen based on the detection limit of chromium by the AA instrument that was used ( 1 to $20 \mathrm{mg} \cdot \ell^{-1}$ ).

Typically, $30 \mathrm{~m} \ell\left(10 \mathrm{mg} \cdot \ell^{-1}\right)$ of the pollutant was passed through the polymers $(300 \mathrm{mg})$. GC-MS, AA and UV measurements were done before and after the spiked water samples have been passed through the polymers. A calibration curve was plotted in order to determine the amount of the spiked pollutants absorbed by the polymers.

\section{Results and discussions}

The results obtained after passing water containing PNP, TCP and chromium through the polymers are summarised in Table 1.

\begin{tabular}{|l|c|c|c|c|c|c|}
\hline \multicolumn{7}{|c|}{ Table 1 } \\
\hline \multirow{2}{*}{ Absolymer } & \multicolumn{7}{|c|}{ Amount of PNP and TCP removed (\%) } \\
\cline { 2 - 7 } & HDI linker & \multicolumn{3}{c|}{ TDI linker } \\
\cline { 2 - 7 } & PNP & TCP & Cr $^{6+}$ & PNP & TCP & Cr $^{6+}$ \\
\hline$\beta$-CD & 64 & 54 & 34 & 68 & 58 & 60 \\
\hline$\beta$-CDMIMOTs & 80 & 69 & 100 & 77 & 69 & 100 \\
\hline$\beta$-CDBUTIMOTs & 68 & 69 & 98 & 64 & 77 & 97 \\
\hline$\beta$-CDPYROTs & 15 & 63 & 100 & 40 & 60 & 95 \\
\hline$\beta$-CDMIMIOD & 65 & 75 & 69 & 55 & 74 & 43 \\
\hline$\beta$-CDBUTIMIOD & 63 & 67 & 51 & 71 & 71 & 95 \\
\hline$\beta$-CDPYRIOD & 24 & 69 & 34 & 48 & 61 & 60 \\
\hline$\beta$-CD: $\beta$-cyclodextrin \\
$\beta$-CDMIMOTs: mono-6-methyl imidazolium tosylate \\
$\beta$-CDBUTIMOTs: mono-6-butyl imidazolium tosylate \\
$\beta$-CDPYROTs: mono-6-pyridinium tosylate \\
$\beta$-CDMIMIOD: mono-6-methyl imidazolium iodide \\
$\beta$-CDPYRIOD: mono-6-pyridinium iodide \\
$\beta$-CDBUTIMIOD: mono-6-butyl imidazolium iodide \\
\hline
\end{tabular}

\section{UV-visible spectroscopy results}

Phenolic compounds are quite prevalent as pollutants in most aquatic systems; PNP was thus selected as a model pollutant. Furthermore, PNP can be analysed by UV spectroscopy since it has chromophores (i.e. covalently bonded but unsaturated groups such as $\mathrm{NO}_{2}, \mathrm{C}=\mathrm{C}$ and $\mathrm{C}=\mathrm{O}$ ) that absorb electromagnetic radiation in the ultraviolet and visible regions of the spectrum (Field et al., 1995). Thus PNP strongly absorbs at $\lambda=318 \mathrm{~nm}$ (visible region of the spectrum). The PNP-spiked water samples that passed through 
the polymers were measured by UV spectroscopy and using the absorbance, the residual PNP concentration was determined using the calibration curve and the results are shown in Table 1 .

It is evident from Table 1 that the CD-IL polymers that were synthesised have generally higher absorption efficiencies for organic pollutants when compared with the native $\beta$-CD/HDI and $\beta$-CD/TDI polymers. For example, absorption of up to $80 \%$ was recorded for the $\beta$-CDMIMOTs/HDI polymer. This is substantially higher than the respective $64 \%$ and $68 \%$ observed for the $\mathrm{CD} / \mathrm{HDI}$ and $\mathrm{CD} / \mathrm{TDI}$ native polymers. It should also be noted that the extraction efficiency of the imidazolium-based polymers is generally superior to those of pyridinium-based polymers. In fact, the extraction efficiency of $15 \%$, which was recorded for $\beta$-CDPYROTs/HDI, is even lower than that of the native polymers.

\section{GC-MS results}

TCP-spiked water samples were passed through the polymers and it was observed that a substantial amount of the TCP was absorbed by the polymers. However, the amount of TCP absorbed by the polymers was found to be less than that absorbed for PNP. These polymers were therefore found to have higher affinity for PNP than TCP (Table 1). This may be attributed to the structure of these 2 compounds; the structure of compounds (compatible geometry) determines the amount of the compound that will fit in the cavity (Linde et al., 2000). TCP is highly branched and could not perfectly pack in the CD cavity while the structure of PNP renders it more amenable to the absorption sites of the $\beta$-CD-IL polymers resulting in higher removal efficiency of PNP than TCP. However, these polymers still showed high absorption efficiencies when compared to the native CD polymers. This implies that the incorporation of the imidazolium ring onto the $\mathrm{CD}$ backbone resulted in an enhancement of absorption efficiency by the polymers.

\section{AAS results}

Besides being readily available in our laboratories, chromium was chosen because it is a toxic inorganic pollutant (heavy metal) especially when present in water as $\mathrm{Cr}^{6+}$. This metal ion can penetrate the skin causing irritation, liver and kidney damage as well as a decrease in male sperm counts (Morales et al., 1999). AAS analysis of the eluant after passing the $\mathrm{Cr}^{6+}$ containing water samples demonstrated that the CD-IL polymers were able to remove $\mathrm{Cr}^{6+}$ with an absorption efficiency of up to $100 \%$. This observation suggests that the IL component incorporated onto the CD backbone was still highly active in the extraction of heavy metals from water. It is noteworthy that the native $\beta$-CD polymers also absorbed $\mathrm{Cr}^{6+}$ from water. Similar studies (Cardas et al., 2005; Brusseau et al., 1997) have also revealed that native $\mathrm{CD}$ polymers indeed form complexes with heavy metal ions. Results for the extraction of $\mathrm{Cr}^{6+}$ using the native polymers as well as the CD-IL polymers are summarised in Table 1 .

\section{Effect of the type of the cation - imidazolium vs. pyridinium}

As shown in Table 1, incorporation of the imidazolium ring onto the $\mathrm{CD}$ backbone generally favours the absorption of both PNP and TCP by the polymers. On the contrary, the pyridinium-based polymers are far much better at the extraction of TCP. Although the IL component also enhances the complexation of $\mathrm{Cr}^{6+}$ ions, due consideration must be given to the type of anion.

\section{Effect of the alkyl chain length attached to the imidazolium ring: methyl vs. butyl}

Previous studies (Visser et al., 2001; Pandey, 2006) suggest that the amount of pollutant absorbed is affected by the length of the alkyl chain attached to the imidazolium ring. These reports stated that the longer the alkyl chain, the less effective the ionic liquid for metal ion extraction. Our investigation seems to support a decrease in the amount of the pollutant absorbed when the alkyl chain is extended. As shown in Table 1, the methylimidazolium polymers were found to possess higher extraction efficiency compared to their butylimidazolium counterparts.

\section{Effect of the type of anion - tosylate vs. iodide}

A study of the effect of the counter-anion (tosylate and iodide) on the absorption efficiency of the polymer revealed no correlation between the amount of organic pollutant (TCP and PNP) absorbed and the type of anion present in the polymer. However, for the absorption of $\mathrm{Cr}^{6+}$, the tosylated polymers had high absorption efficiencies compared to the iodinated polymers. The tosylate anion is hydrophobic and thus enhances the chelation (hexavalent dentition) of the chromium with the cationic component of the CD-IL polymers. The tosylate anion

\begin{tabular}{|c|c|c|c|c|c|c|c|c|c|c|}
\hline \multicolumn{11}{|c|}{$\begin{array}{c}\text { Table } 2 \\
\text { Comparison of surface area vs. percentage absorption }\end{array}$} \\
\hline \multirow[t]{2}{*}{ Sample } & \multicolumn{5}{|c|}{ HDI } & \multicolumn{5}{|c|}{ TDI } \\
\hline & $\begin{array}{c}\text { Surface } \\
\text { area } \\
\left(\mathrm{m}^{2} / \mathrm{g}\right)\end{array}$ & $\begin{array}{l}\text { \% PNP } \\
\text { absorbed }\end{array}$ & $\begin{array}{c}\text { \%TCP } \\
\text { absorbed }\end{array}$ & $\begin{array}{l}\% \mathrm{Cr}^{6+} \\
\text { absorbed }\end{array}$ & $\begin{array}{c}\% \mathrm{Cd}^{2+} \\
\text { absorbed }\end{array}$ & $\begin{array}{c}\text { Surface } \\
\text { area } \\
\left(\mathrm{m}^{2} / \mathrm{g}\right)\end{array}$ & \begin{tabular}{c|c}
$\%$ PNP \\
absorbed
\end{tabular} & $\begin{array}{c}\text { \%TCP } \\
\text { absorbed }\end{array}$ & $\begin{array}{c}\% \mathrm{Cr}^{6+} \\
\text { absorbed }\end{array}$ & $\begin{array}{l}\% \mathrm{Cd}^{2+} \\
\text { absorbed }\end{array}$ \\
\hline$\beta-\mathrm{CD}$ & 2.53 & 64 & 54 & 34 & 18 & 1.706 & 68 & 58 & 60 & 24 \\
\hline$\beta$-CDMIMOTs & 23.26 & 80 & 69 & 100 & 33 & 22.71 & 77 & 69 & 100 & 28 \\
\hline$\beta$-CDBUTIMOTs & 2.99 & 68 & 69 & 98 & 32 & 10.51 & 64 & 77 & 97 & 23 \\
\hline$\beta$-CDPYROTs & 2.89 & 15 & 63 & 100 & 24 & 1.64 & 40 & 60 & 95 & 20 \\
\hline$\beta$-CDMIMIOD & 2.51 & 65 & 75 & 69 & 15 & 1.81 & 55 & 74 & 43 & 18 \\
\hline$\beta$-CDBUTIMIOD & 10.88 & 63 & 67 & 51 & 21 & 1.78 & 71 & 71 & 95 & 17 \\
\hline$\beta$-CDPYRIOD & 0.4427 & 24 & 69 & 34 & 10 & 7.00 & 48 & 61 & 60 & 13 \\
\hline
\end{tabular}



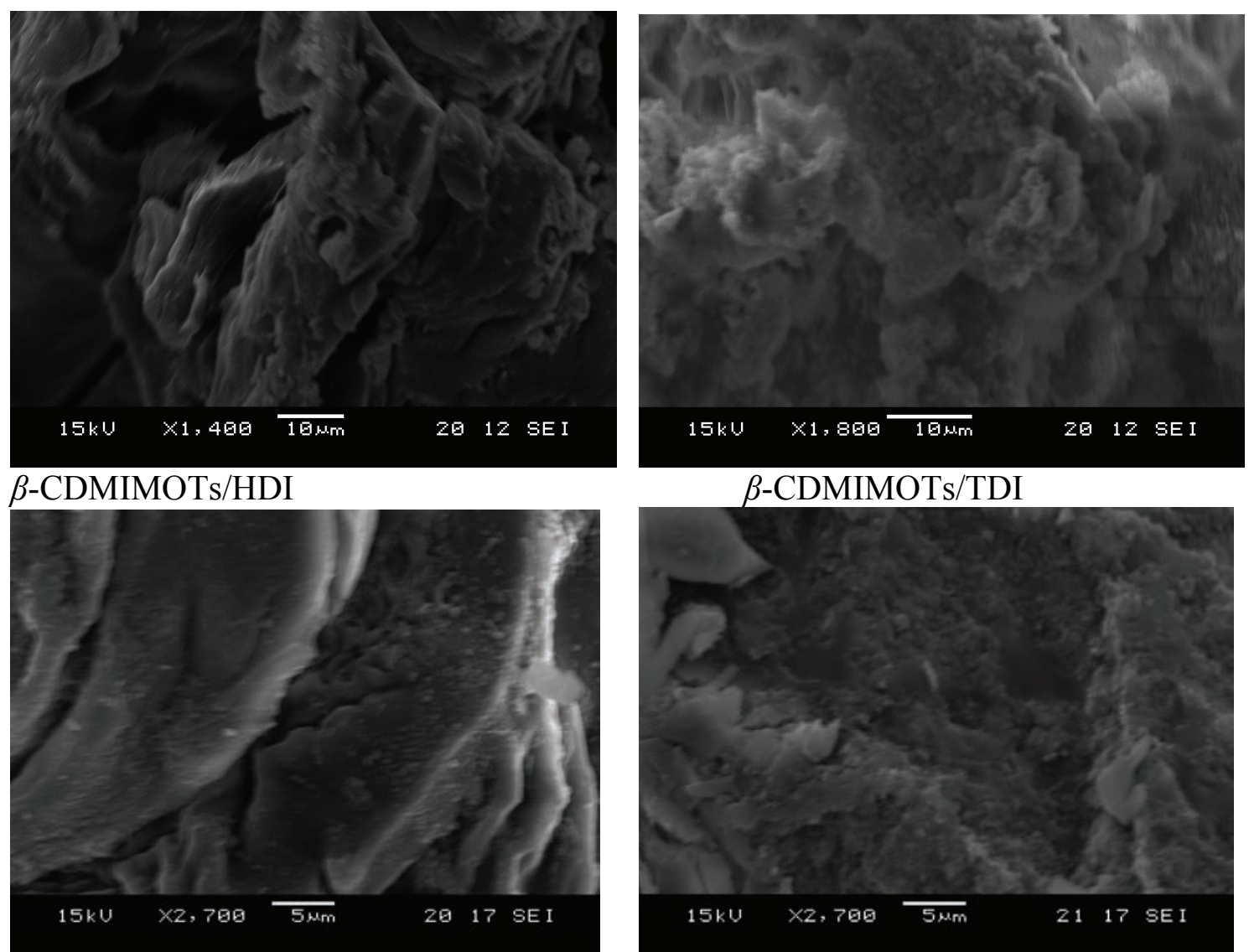

$\beta$-CDMIMIOD/TDI

\section{$\beta$-CDMIMOD/TDI}

Figure 3

SEM micrographs of the $\beta-C D-I L$ polymers

enhances the chelation of the heavy metal by repelling the water molecules from the cationic component. The iodide anion is, on the other hand, a hydrophilic anion and has the opposite effect. This observation is in agreement with reports by Welton (1999) and Cocalia et al. (2006) which reported a high absorption efficiency of heavy metal ions when hydrophobic anions were used. Hydrophobic anions repel water molecules thus creating an environment conducive for IL-metal-ion complex formation.

\section{Comparison of absorption vs. surface area (BET results)}

After determining the absorption efficiencies of the polymers, it was necessary to assess whether there was any correlation between the amount of pollutant absorbed and the surface area of the polymers. The surface analysis data (Table 2) do not reveal any direct relationship between the amount of pollutant absorbed and the surface areas of the polymers. For example, both $\beta$-CDMIMOTs/HDI and $\beta$-CDPYROTs/HDI polymers showed $100 \%$ absorption efficiency for PNP, yet their surface areas were significantly different $\left(23.26 \mathrm{~m}^{2} \cdot \mathrm{g}^{-1}\right.$ and $2.89 \mathrm{~m}^{2} \cdot \mathrm{g}^{-1}$, respectively).

\section{Scanning electron microscopy (SEM)}

Scanning electron microscopy was carried out to determine the surface morphology of the polymers. This was done in order to determine whether the absorption efficiency of the polymers could be linked to the surface morphology of the polymers. The morphology of the polymers was found to have no bearing on the amount of the pollutant absorbed by the polymers. Although the polymers had different morphological appearances (see Fig. 3), their absorption efficiencies were still comparable. For example, although $\beta$-CDMIMOTs/ TDI and $\beta$-CDMIMOTs/HDI appear different under a scanning electron microscope, they both absorbed $100 \%$ of $\mathrm{Cr}^{6+}$. However, the same polymers show different absorption capabilities for PNP. $\beta$-CDMIMOTs/TDI absorbed $77 \%$ of the model pollutant while $\beta$-CDMIMOTs/HDI exhibited an absorption efficiency of $80 \%$.

\section{Conclusion}

Paranitrophenol, 2,4,6-trichlorophenol and $\mathrm{Cr}^{6+}$ were successfully extracted from an aqueous mixture using CD-IL. A high percentage removal by these ionic liquid polymers was observed after passing water containing the model pollutants through the polymers.. The polymers showed fairly high absorption efficiencies for both the inorganic $\left(\mathrm{Cr}^{6+}\right)$ and organic pollutants (PNP and TCP). CD-IL polymers can potentially be used for the removal of both organic and inorganic pollutants from drinking water systems.

\section{Acknowledgements}

Financial assistance from the University of Johannesburg and the National Research Foundation (NRF) is appreciated. 


\section{References}

BENDER ML and KOMIYANA M (1978) Cyclodextrin Chemistry. Springer-Verlag Berlin, Heidelberg, New York. 1-3.

BRUSSEAU ML, WANG X and WANG W-Z (1997) Simultaneous elution of heavy metals and organic compounds from soil by cyclodextrin. Environ. Sci. Technol. 31 1087-1092.

CARDAS S, FORAY M-F, GADELLE A. GERBAUD G and BARDET M (2005) High-resolution solid state ${ }^{13} \mathrm{C}$ NMR study of per(3,6-anhydro)- $\alpha$-cyclodextrin based polymers and of their chromium complexes. Carbohydr. Polym. 61 88-94.

CRUZ DMP (2000) Thermal Analysis and Oxidation Reactions with Room-Temperature Ionic Liquids. DOE ERULF Program, University of Puerto Rico, National Renewable Energy Laboratory, Golden, Colorado.

FERREIRA P, GONCALVES IS, PILLINGER M, ROCHA J, SANTOS P and TEIXEIRA-DIAS JJC (2000) Modifications of $\beta$-cyclodextrin with ferrocenyl groups by ring opening of an encapsulated [1] ferrocenophane. Organomet. 19 1455-1455.

FIELD LD, STERNHELL S and KALMAN JR (1995) Organic Structures from Spectra. John Wiley and Sons, New York. p 6.

GAO XM, TONG LH, INOUOE Y and TAI A (1995) Synthesis and characterisation of novel multifunctional host compounds. 4 cyclodextrin derivatives bearing chromophores. Synth. Commun. $25703-710$.

LEWIS R and EVANS W (2001) Chemistry (2 $2^{\text {nd }}$ edn.). 415 pp.

LI D and MA M (1999) Nanosponges: From inclusion chemistry to water purifying technology. Chem. Tech. 35 31-37.

LINDE JJ, FREESE SD and PIETERSE S (2000) Evaluation of Powdered Activated Carbon (PAC) for the Removal of Taste and Odour Causing Compounds from Water. WRC Report No.1124/1/04. Water Research Commission, Pretoria, South Africa.

LIU J, JIANG G, CHI Y, CAI Y, ZHOU Q and HU J-T (2003) Use of ionic liquids for liquid-phase microextraction of polycyclic hydrocarbons. Anal. Chem. 75 5870-5876.

LIU J, LI N, JIANG G, LIU J, JOHNSSON JA and WEN M (2005) Disposable ionic liquid coating for headspace solid-phase microextraction of benzene, toluene, ethylbenzene, and xylenes in paints by gas chromatography-flame ionization detection. J. Chromatogr. A. 1066 27-32.

MAMBA BB, KRAUSE RW, MALEFETSE TJ and NXUMALO EN (2007) Monofunctionalised cyclodextrin polymers for the removal of organic pollutants from water. Environ. Chem. Lett. 5 79-84.

MAMBA BB, KRAUSE RW, MALEFETSE TJ, MHLANGA SD, SALIPIRA KL, SITHOLE SP, and NXUMALO EN (2007) Removal of geosmin and 2-methylisorboneol (2-MIB) in water from Zuikerbosch Treatment Plant (Rand Water) using $\beta$-cyclodextrin polyurethanes. Water $S A 33$ (2) 223-228.

MHLANGA SD, MAMBA BB, KRAUSE RW and TJ MALEFETSE (2007) Removal of organic contaminants from water using nanosponge cyclodextrin polyurethanes. J. Chem. Technol. Biotechnol. 82 382-388.

MORALES MM, MARTI P, LLOPIS A, CAMPOS L and SAGRADO S (1999) An environmental study by factor analysis of surface seawaters in the Gulf of Valencia (Western Mediterranean). Anal. Chim. Acta 394 109-117.

OLESZCZUK P and BARAN S (2004) Application of solid-phase extraction to determination of polycyclic aromatic hydrocarbons in sewage sludge extracts. J. Hazard. Mater. 113 237-245.

PANDEY S (2006) Analytical applications of room temperature ionic liquids: A review of recent efforts. Anal. Chim. Acta 556 38-45.

PEREIRO AB, LEGIDO JL and RODRIGUEZ A (2007) Physical properties of ionic liquids based on 1-alkyl-3-methylimidazolium cation and hexafluorophosphate as anion and temperature dependence. J. Chem. Thermodyn. 39 1219-1230.

SALIPIRA KL, MAMBA BB, KRAUSE RW, MALEFETSE TJ and DURBACH SH (2007) Carbon nanotubes and cyclodextrin polymers for the removal of organic pollutants from drinking water. Environ. Chem. Lett. 513-17.

SZEITJLI J (1998) Introduction and general overview of cyclodextrin chemistry. Chem. Rev. 98 1743-1753.

US ENVIRONMENTAL PROTECTION AGENCY (EPA) (2000) Chemical Precipitation, US EPA, Washington DC. (EPA832-f-00-018).

VISSER AE, SWATLOSKI RP, GRIFFIN ST, HARTMEN DH and ROGERS RD (2001) Liquid extraction of metal ions in Room temperature ionic liquids. Sep. Sci. Technol. 36 785-804.

VISSER AE, SWATLOSKI RP, REICHERTWM, MAYTON R, SHEFF S, WIERZBICKI A, DAVIS JH and ROGERS RD (2002) Task-Specific ionic liquids incorporating novel cations for the coordinating and extraction of $\mathrm{Hg}^{2+}$ and $\mathrm{Cd}^{2+}$ : Synthesis, Characterization, and Extraction Studies. Environ. Sci. Technol. 36 2523-2529.

WEI G-T, YANG Z and CHEN C-J (2003) Room temperature ionic liquid as a novel medium for liquid/liquid extraction of heavy metals. Anal. Chim. Acta 488 183-192.

WELTON T (1999) Room-Temperature ionic liquid. Solvents for synthesis and catalysis. Chem. Rev. 99 2071-2083.

ZHONG N, BYUN H-S and BITTMAN R (1998) An improved synthesis of 6-O-monotosyl-6-deoxy- $\beta$-cyclodextrin. Tetrahedron Lett. 39 2919-2920. 